\title{
Cyanobactéries des rivières Boubo et Mé dans le Sud côtier de la Côte d'Ivoire
}

\author{
Moreto SALLA $^{1 *}$, Philippe Da KOUHETE ${ }^{1}$, Sébastien Ouffoué KOFFI ${ }^{2}$, et \\ Dossahoua TRAORE ${ }^{1}$ \\ ${ }^{1}$ Laboratoire de Botanique, U.F.R. Biosciences, Université de Cocody, 22 B.P. 582 Abidjan 22, Côte d'Ivoire. \\ ${ }^{2}$ Centre Ivoirien Anti-Pollution (C.I.A.POL.), 20 B.P. 650 Abidjan 20, Côte d'Ivoire. \\ *Auteur correspondant, E-mail : salla.moreto@yahoo.fr; Tel : (+225) 07785522 /(+225) 02275520
}

\section{RESUME}

Vingt sept taxa de Cyanoprokaryota, répartis entre les ordres des Chroococcales et des Hormogonales, ont été récoltés entre mai 2009 et février 2010 dans les rivières côtières Boubo et Mé dans le Sud de la Côte d'Ivoire. Neuf de ces taxa sont nouveaux pour ce pays. Des données morphologiques et de mensurations de ces taxa sont fournies ainsi que leur répartition phytogéographique au niveau mondial et en Afrique où ces taxa ont déjà été signalés. Leur distribution au niveau des stations de récolte a été aussi donnée. Des illustrations sont fournies pour chaque taxon cité.

(C) 2011 International Formulae Group. All rights reserved.

Mots clés : Cyanoprokaryota, Chroococcales, Hormogonales, rivières Boubo et Mé, Côte d'Ivoire.

\section{INTRODUCTION}

L'eau douce, indispensable à la vie et à la santé humaine, est une ressource limitée en Afrique, particulièrement dans la zone subsaharienne. Selon l'Organisation Mondiale de la Santé (OMS), parmi les menaces qui touchent les eaux douces, on peut considérer la contamination par les cyanobactéries et leurs toxines comme un risque émergent limitant les usages de cette ressource (Chorus et Bartram, 1999). De plus la prolifération du phytoplancton en général et celle des cyanobactéries en particulier peut entraîner l'eutrophisation des lacs et rivières.

Les Cyanoprokaryota de l'Afrique Occidentale ont été relativement peu étudiées, notamment par Berger et al. (2005) au Sénégal, Cecchi et al. (2005) au Burkina Faso,
Roger et Reynaud (1977) au Sénégal, Serpette (1955) en Afrique Occidentale, Whittton (1968) en Sierra Leone. La plupart de ces travaux sont d'ordre écologique et peu portent sur l'étude systématique.

En Côte d'Ivoire les seuls travaux réalisés exclusivement sur les Cyanoprokaryota sont de Bourrelly (1961), grâce aux récoltes provenant du marais de l'Agnéby, d'une mare de Divo, de l'étang du Banco, du bassin d'Adiopodoumé et de la lagune Ébrié au niveau de Bingerville. Il a observé vingt neuf taxa pour la plupart cosmopolites. Des travaux systématiques sur les Cyanoprokaryota des milieux lotiques sont inexistants. Néanmoins des études sur le phytoplancton des eaux duçaquicoles lotiques faisant cas des Cyanoprokaryota ont été 
réalisées par Iltis (1982), Ouattara et al. (2000), Niamien (2010). Notre connaissance des Cyanoprokaryota de Côte d'Ivoire reste donc très fragmentaire et limitée. Dès lors il nous paraît utile de publier ici nos observations sur les Cyanoprokaryota de quelques récoltes effectuées dans les rivières côtières Boubo et Mé au Sud de la Côte d'Ivoire.

\section{MATERIEL ET METHODES}

\section{Présentation du milieu d'étude}

Le milieu d'étude est localisé dans la partie méridionale de la Côte d'Ivoire. Il est situé entre $3^{\circ} 45^{\prime}$ et $5^{\circ} 30^{\prime}$ de latitude Nord, $5^{\circ} 15^{\prime}$ et $6^{\circ} 20^{\prime}$ de longitude Ouest. Les rivières Boubo et Mé, qui sont les cours d'eau de prélèvement pour cette étude, ont respectivement des superficies réceptrices de $4702 \mathrm{~km}^{2}$ et $2458 \mathrm{~km}^{2}$. Sur chaque cours d'eau, trois stations sont définies en fonction de leur accessibilité (Figure 1).

Ainsi, sur les deux rivières, les valeurs moyennes de température, de conductivité et de $\mathrm{pH}$ sont données de l'amont en aval comme suit :
* Sur la rivière Boubo, on trouve les stations de :

- Divo $\left(5^{\circ} 75^{\prime} \mathrm{N}\right.$ et $\left.5^{\circ} 31^{\prime} \mathrm{W}\right)$ située au niveau du pont sur l'axe Divo-Lakota, avec comme moyennes: de température $(25,70$ $\left.{ }^{\circ} \mathrm{C}\right)$, de conductivité $\left(122,36 \mu \mathrm{S} . \mathrm{cm}^{-1}\right)$, de $\mathrm{pH}(6,97)$.

- PALMCI-Boubo $\left(5^{\circ} 61^{\prime} \mathrm{N}\right.$ et $5^{\circ} 25^{\prime}$ W) située à l'aval de l'huilerie de la société PALMCI de Divo où l'on a comme moyennes : de température $\left(25,95{ }^{\circ} \mathrm{C}\right)$, de conductivité $\left(113,45 \mu{\left.\mathrm{S} . \mathrm{cm}^{-1}\right)}^{-1}\right.$, de $\mathrm{pH}(7,28)$.

- Adahidougou $\left(5^{\circ} 18^{\prime} \mathrm{N}\right.$ et $\left.5^{\circ} 11^{\prime} \mathrm{W}\right)$ située au niveau du pont sur l'axe GrandLahou-Fresco, avec comme moyennes: de température $\left(26,00{ }^{\circ} \mathrm{C}\right)$, de conductivité (293,7 $\mu$ S.cm $\left.{ }^{-1}\right)$, de $\mathrm{pH}(6,68)$.

* Sur la rivière Mé il y a les stations de :

- Abou $\left(5^{\circ} 72^{\prime} \mathrm{N}\right.$ et $\left.3^{\circ} 68^{\prime} \mathrm{W}\right)$ située sur l'axe Adzopé-Yakassé-Attobro, avec comme moyennes: de température $\left(25,00{ }^{\circ} \mathrm{C}\right)$, de conductivité $\left(133,9 \mu \mathrm{S} . \mathrm{cm}^{-1}\right)$, de $\mathrm{pH}(6,89)$.

- Mafou, un affluent de la Mé $\left(5^{\circ} 70^{\prime} \mathrm{N}\right.$ et $3^{\circ} 77^{\prime} \mathrm{W}$ ) située au niveau du pont sur l'axe Azaguié-Adzopé, avec comme moyennes : de température $\left(25,83{ }^{\circ} \mathrm{C}\right)$, de conductivité $(78,5$ $\left.\mu \mathrm{S} . \mathrm{cm}^{-1}\right)$, de $\mathrm{pH}(6,92)$.

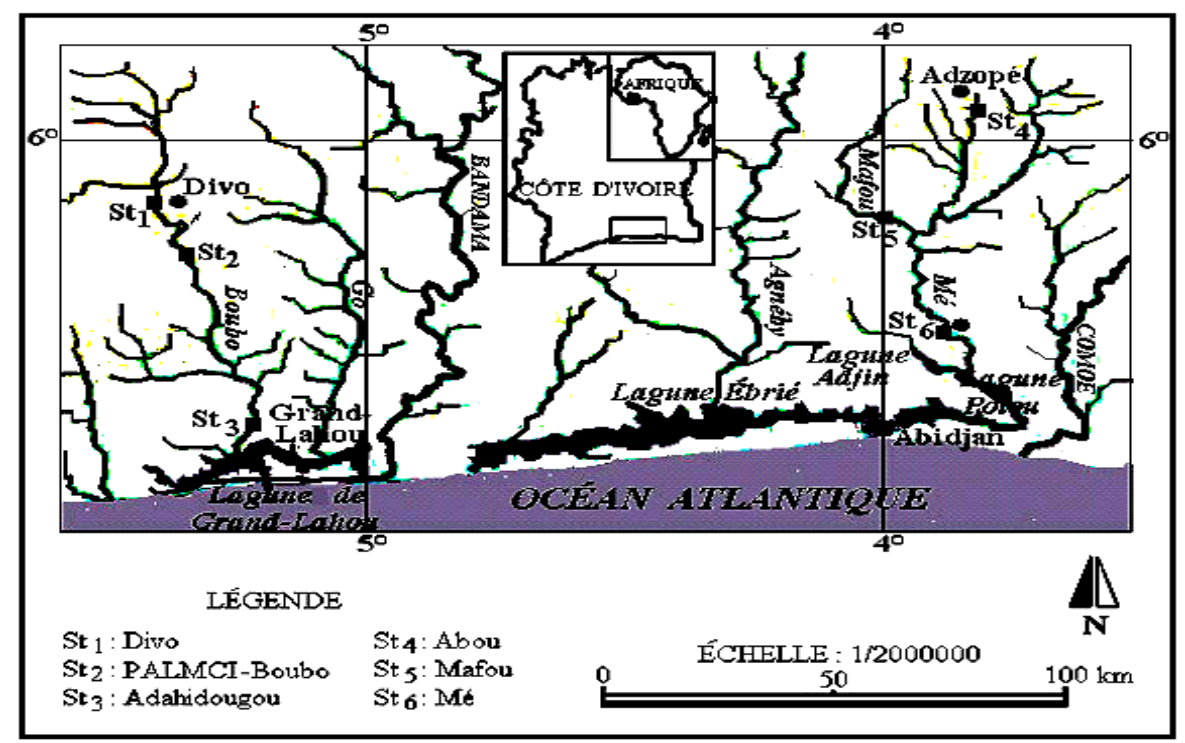

Figure 1 : Localisation des stations d'échantillonnage sur les rivières Boubo et Mé. 
- Mé $\left(5^{\circ} 32 \mathrm{~N}\right.$ et $\left.3^{\circ} 67^{\prime} \mathrm{W}\right)$ située au niveau du pont sur l'axe Abidjan-Alépé avec comme moyennes: de température $(25,43$ $\left.{ }^{\circ} \mathrm{C}\right)$, de conductivité $\left(5,07 \mu \mathrm{S} . \mathrm{cm}^{-1}\right)$, de $\mathrm{pH}(6,72)$.

\section{Récolte des échantillons}

Les échantillons ont été récoltés à l'aide d'un filet à plancton de $20 \mu \mathrm{m}$ de vide de maille et fixés au formol du commerce à la concentration finale de 5 pc. Les sédiments obtenus dans les piluliers de $40 \mathrm{ml}$ au bout de quelques jours sont montés entre lame et lamelle pour observation sur un microscope optique de type Olympus équipé d'un appareil photographique numérique.

Les facteurs physico-chimiques ont été mesurés in situ grâce à un pHmètre $(\mathrm{HACH}$ EC 10) pour le $\mathrm{pH}$ et un conductimètre (HACH CO 150) pour la conductivité $(\mu \mathrm{S}$. $\left.\mathrm{cm}^{-1}\right)$, la température $\left({ }^{\circ} \mathrm{C}\right)$ et la salinité $(\mathrm{pm})$. Les appareils sont d'abord mis sous tension avant la plongée de la sonde pour un affichage numérique des valeurs. Les sels minéraux ont été dosés au laboratoire par spectrophotométrie à UV visibles.

\section{RESULTATS}

\section{Description des taxons observés}

La classification suivie est celle proposée par Komárek et Anagnostidis (1999 et 2005). Dans chaque ordre, les genres, les espèces et les taxa spécifiques sont cités par ordre alphabétique. Les taxa marqués d'un astérisque $\left(^{*}\right)$ sont nouveaux pour la flore algale ivoirienne. Les traits d'échelle des illustrations représentent $20 \mu \mathrm{m}$.

\section{Cyanoprokaryota - Cyanophyceae - Chroococcales \\ *Aphanocapsa grevillei (Hassall) Rabenhorst \\ Colonies sphériques; cellules de 3-5 $\mu \mathrm{m}$ de diamètre, solitaires ou groupées dans une gelée homogène, incolores (Fig. 2).}

Répartition phytogéographique : cosmopolite. En Afrique: Burkina Faso, Burundi. Lieu de récolte: Divo, Adahidougou.

*Aphanothece castagnei (Brébisson) Rabenhorst

Colonies mucilagineuses, irrégulières, cellules sphériques à ellipsoïdales de 4,5-6,7 x 3-4 $\mu \mathrm{m}$, dépourvues de pseudovacuoles gazeuses (Fig. 3).

Répartition phytogéographique : cosmopolite. En Afrique: Burundi. Lieu de récolte : PALMCI-Boubo.

Chroococcus dispersus (Keissler) Lemmermann

Cellules plus ou moins sphériques de 3,7-4,9 $\mu \mathrm{m}$ de diamètre, groupées sans forme définie, avec un contenu granuleux (Fig. 4).

Répartition phytogéographique : cosmopolite. En Afrique: Burkina Faso, Burundi, Côte d'Ivoire. Lieu de récolte: Adahidougou.

\section{Chroococcus minutus (Kützing) Nägeli}

Colonies de 4 cellules; cellules de 45,4 $\mu \mathrm{m}$ de diamètre (Fig. 5). Répartition phytogéographique: cosmopolite. En Afrique: Afrique du Sud, Algérie, Angola, Burkina Faso, Cameroun, Côte d'Ivoire, Gabon, Kenya, Libye, Madagascar, Maroc, Ouganda, République Démocratique du Congo, São-Tomé et Principe, Tchad, Tunisie, Zambie. Lieu de récolte : Adahidougou.

\section{Chroococcus turgidus (Kützing) Nägeli}

Colonies de 1-4 grosses cellules entourées d'une gaine gélatineuse ; cellules de 14,5-18,5 $\mu \mathrm{m}$ de diamètre (Fig. 6).

Répartition phytogéographique: cosmopolite. En Afrique: Afrique du Sud, Algérie, Angola, Burkina Faso, Cameroun, Côte d'Ivoire, Égypte, Gabon, Kenya, Libye, Madagascar, Malawi, Maroc, Mozambique, République Démocratique du Congo, SãoTomé et Principe, Sénégal, Soudan, Tanzanie, Tchad, Tunisie, Zambie. Lieu de récolte : Adahidougou. 


\section{Eucapsis alpina Clements \& Schantz}

Colonies cubiques de 32 à 125 cellules, composées de petites colonies cubiques de 8 cellules; cellules de 5-9,4 $\mu \mathrm{m}$, réunies dans une gaine mucilagineuse (Fig. 7).

Répartition phytogéographique : cosmopolite. En Afrique: Côte d'Ivoire, Gabon. Lieu de récolte : Adahidougou.

*Gloeocapsa itzigsohnii Bornet

Colonies régulières ou irrégulières de cellules regroupées 4 à 4 ; cellules de 3-4 $\mu \mathrm{m}$ de diamètre (Fig. 8).

Répartition phytogéographique : cosmopolite. En Afrique : mal connue. Lieu de récolte : PALMCI-Boubo.

Microcystis aeruginosa (Kützing) Kützing

Colonies irrégulières ; cellules sphériques de 5,3-7,8 $\mu \mathrm{m}$ de diamètre, pourvues de pseudovacuoles gazeuses (Fig. 9).

Répartition phytogéographique : cosmopolite. En Afrique : Burkina Faso, Côte d'Ivoire. Lieu de récolte: Adahidougou, PALMCI-Boubo.

*Microcystis holsatica Lemmermann

Colonies sphériques ou irrégulières ; cellules de 0,6-1,3 $\mu \mathrm{m}$ de diamètre (Fig. 10). Répartition phytogéographique : cosmopolite. En Afrique: Afrique du Sud, Kenya, Ouganda, République Démocratique du Congo, Soudan, Tchad. Lieu de récolte : Adahidougou.

\section{Synechocystis aeruginosus Nägeli}

Amas de cellules solitaires, libres, sphériques de 5,5-7,2 $\mu \mathrm{m}$ de diamètre (Fig. 11). Répartition phytogéographique: cosmopolite. En Afrique : Côte d'Ivoire. Lieu de récolte : Divo, PALMCI-Boubo.

\section{Cyanoprokaryota - Cyanophyceae - Hormogonales \\ Anabaena affinis Lemmermann}

Trichomes droits, avec des cellules sphériques de 4,1-6 x 5-6 $\mu \mathrm{m}$; hétérocytes subsphériques de 7,8-9,3 x 6,7-7,5 $\mu \mathrm{m}$ de diamètre (Fig. 12).

Répartition phytogéographique : subcosmopolite. En Afrique : Côte d'Ivoirte. Lieu de récolte : Divo, PALMCI-Boubo.

\section{Anabaena spiroides Klebs}

Trichomes enroulés; cellules de 5-6,3 $\mathrm{x} \quad 5-5,6 \mu \mathrm{m}$ de diamètre; hétérocytes subsphériques de 7-8 $\mu \mathrm{m}$ de diamètre (Fig. 13).

Répartition phytogéographique: cosmopolite. En Afrique: Afrique du Sud, Côte d'Ivoire, Égypte, Ouganda, République Démocratique du Congo, Tchad. Lieu de récolte : Adahidougou.

\section{Anabaena sp.}

Trichomes droits, avec des cellules sphériques de 7-9,5 x 13,8-14,5 $\mu \mathrm{m}$; hétérocytes subsphériques de 11-12 x 11,512,3 $\mu \mathrm{m}$ de diamètre (Fig. 14). Lieu de récolte : PALMCI-Boubo.

*Lyngbya major Gomont

Filaments droits ou courbés, de 12,215,6 $\mu \mathrm{m}$ de diamètre, pourvus d'une gaine ferme ; trichomes de 11,8-14 $\mu \mathrm{m}$ de diamètre, non rétrécis aux articulations ; cellules de 2,2$3 \mu \mathrm{m}$ de longueur (Fig. 15).

Répartition phytogéographique : subcosmopolite. En Afrique : Afrique du Sud, Algérie, Égypte, Ouganda, République

Démocratique du Congo, Tchad, Tunisie. Lieu de récolte : PALMCI-Boubo.

\section{Nostoc entophytum Bornet \& Flahault}

Thalles microscopiques, punctiformes ; trichomes de 2,5-6 x 5,3-6 $\mu \mathrm{m}$ de diamètre ; hétérocytes subsphériques de 7,5-9 x 6,4-7 $\mu \mathrm{m}$ de diamètre (Fig. 16).

Répartition phytogéographique :

subcosmopolite. En Afrique: Côte d'Ivoire,Tchad. Lieu de récolte : Divo.

\section{* Oscillatoria acuta Bruhl. \& Biswas}

Trichomes de 3,9-4,4 $\mu \mathrm{m}$ de diamètre, atténués aux extrémités, non rétrécis aux articulations; articles aussi longs que larges ; articles terminaux coniques, atténués, généralement aigus, de 2,7-5,6 $\mu \mathrm{m}$ de longueur (Fig. 17).

Répartition phytogéographique: paléotropicale. En Afrique: Tchad. Lieu de récolte : Divo.

\section{* Oscillatoria chlorina Kützing ex Gomont}

Trichomes de 6,2-7,4 $\mu \mathrm{m}$ de diamètre, non rétrécis aux articulations; articles longs de 4,3 $\mu \mathrm{m}$ (Fig. 18).

Répartition phytogéographique: cosmopolite. En Afrique: Afrique du Sud, 
Sud-Ouest Africain, Tanzanie, Tchad. Lieu de récolte : Adahidougou.

Oscillatoria jenensis G. Schmidle

Trichomes de 21-24,3 $\mu \mathrm{m}$ de diamètre, apex atténué et recourbé ; articles courts, de 4,2-6,3 $\mu \mathrm{m}$ de longueur, à peine rétrécis au niveau des articulations (Fig. 19).

Répartition phytogéographique : cosmopolite. En Afrique : Côte d'Ivoire. Lieu de récolte : Divo.

\section{"Oscillatoria mougeotii (Kützing) \\ Lemmermann}

Trichomes de 10,4-11,2 $\mu \mathrm{m} \quad \mathrm{de}$ diamètre, non rétrécis aux niveau des articulations ; articles courts, de 2-4 $\mu \mathrm{m}$ de longueur, pourvus de pseudovacuoles gazeuses (Fig. 20).

Répartition phytogéographique : subcosmopolite. En Afrique: Maroc, Mozambique, République Démocratique du Congo, Tchad. Lieu de récolte : Adahidougou. Oscillatoria ornata Gomont

Trichomes de 10-11,2 $\mu \mathrm{m}$ de diamètre, rétrécis au niveau des articulations; articles courts de 4,1-4,8 $\mu \mathrm{m}$ de longueur (Fig. 21).

Répartition phytogéographique : cosmopolite. En Afrique: Côte d'Ivoire, Gabon, Libye, Ouganda, Tchad. Lieu de récolte : PALMCI-Boubo.

\section{Oscillatoria princeps Vaucher ex Gomont}

Trichomes de 22-23,5 $\mu \mathrm{m}$ de diamètre, légèrement rétrécis au niveau des articulations ; articles très courts, de 2,4-4,5 $\mu \mathrm{m}$ de longueur (Fig. 22).

Répartition phytogéographique : cosmopolite. En Afrique: Afrique du Sud, Algérie, Burundi, Côte d'Ivoire, Gabon, Guinée Conakry, Kenya, Libye, Madagascar, Mozambique, Ouganda, République Centrafricaine, République Démocratique du Congo, Rwanda, São Tomé et Principe, Sénégal, Sierra Leone, Soudan, Tanzanie, Tchad, Tunisie. Lieu de récolte: Adahidougou, PALMCI-Boubo.

\section{Oscillatoria sancta (Kützing) Gomont}

Trichomes de 10,3-11,4 $\mu \mathrm{m} \quad \mathrm{de}$ diamètre, rétrécis au niveau des articulations ; articles très courts, de 3-4,5 $\mu \mathrm{m}$ de longueur (Fig. 23).

Répartition phytogéographique : cosmopolite. En Afrique: Afrique du Sud,
Algérie, Burundi, Cameroun, Côte d'Ivoire, Kenya, Malawi, Maroc, République Démocratique du Congo, Sierra Leone, Tanzanie, Tchad, Zambie. Lieu de récolte: Adahidougou, PALMCI-Boubo.

"Phormidium simplissimum (Gomont) Anagnostidis \& Komárek

[syn. : Oscillatoria simplissima Gomont] Trichomes de 6-7 $\mu \mathrm{m}$ de diamètre, non rétrécis au niveau des articulations; articles très courts, de 2-3 $\mu \mathrm{m}$ de longueur (Fig. 24).

Répartition phytogéographique : pantropicale. En Afrique : Burundi, Tanzanie, Zambie. Lieu de récolte : Divo.

\section{Plectonema gracillimum Hansgirg}

Trichomes de 2,2-3,5 $\mu \mathrm{m}$ de diamètre ; articles de 6-10,6 $\mu \mathrm{m}$ de longueur, pris dans une gaine mince et ferme (Fig. 25).

Répartition phytogéographique : cosmopolite. En Afrique : Côte d'Ivoire. Lieu de récolte : Adahidougou, Mafou, Mé.

\section{Pseudanabaena crassa Vozzhennikova}

Trichomes flexibles en chapelet de cellules; cellules de 3,4-6,3 x 7-4,5 $\mu \mathrm{m}$ (Fig. 26).

Répartition phytogéographique: cosmopolite. En Afrique : Côte d'Ivoire. Lieu de récolte: Adahidougou, Divo, PALMCIBoubo.

Spirulina princeps W. \& G.S. West

Trichomes de 3,3-4,6 $\mu \mathrm{m}$ de diamètre, régulièrement spiralés ; spires de 9,3-10,6 $\mu \mathrm{m}$ de diamètre (Fig. 27).

Répartition phytogéographique : cosmopolite. En Afrique : Côte d'Ivoire. Lieu de récolte : Divo.

Tychonema bornetii (Zukal) Anagnostidis \& Komárek

[syn. : Oscillatoria bornetii (Zukal) Geitler]

Trichomes droits de 13,8-14,7 $\mu \mathrm{m}$ de diamètre, articles non rétrécis aux articulations, de 3-4 $\mu \mathrm{m} \quad$; protoplasme granuleux (Fig. 28).

Répartition phytogéographique : cosmopolite. En Afrique: Côte d'Ivoire, Tchad, Zambie. Lieu de récolte: Adahidougou, PALMCI-Boubo. 




2

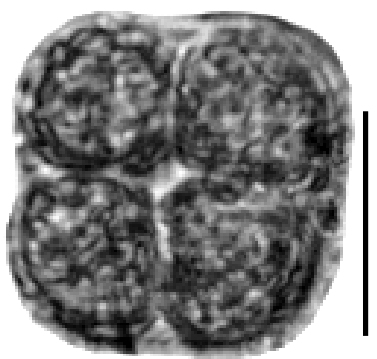

6

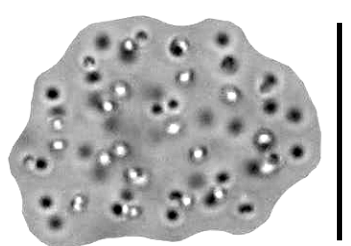

10

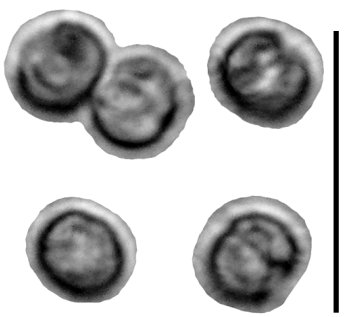

11

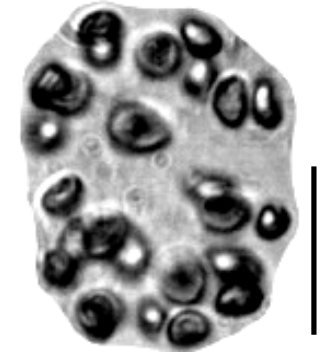

3

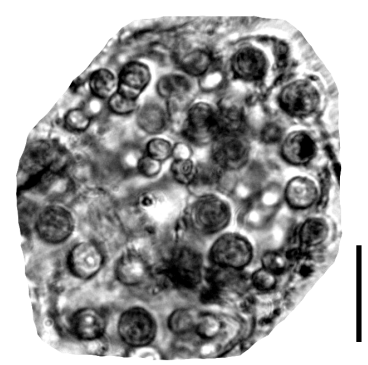

7

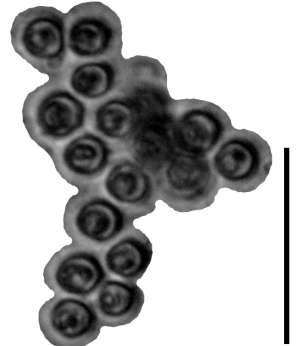

4

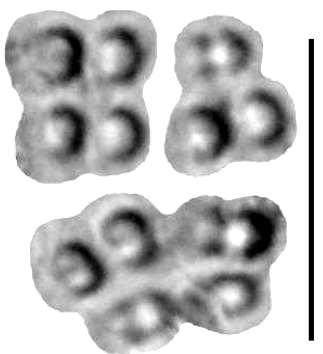

8

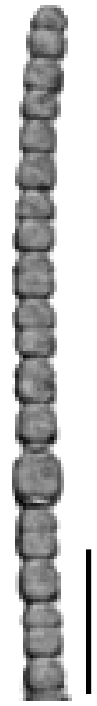

12

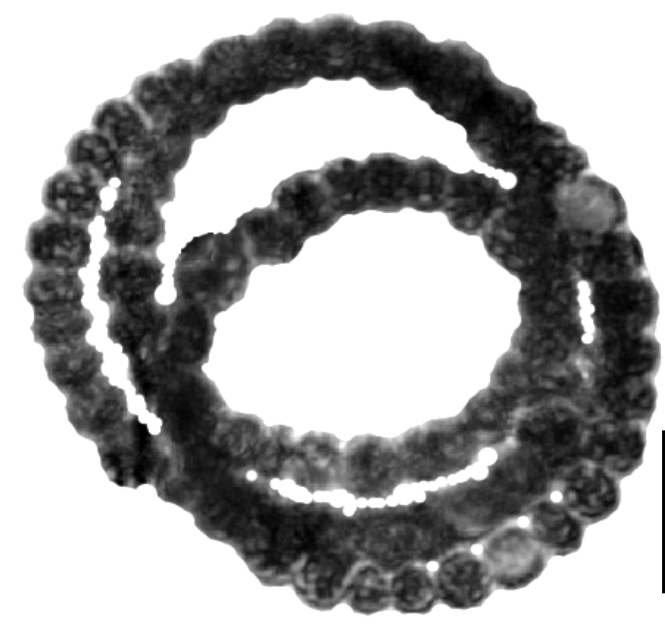

13

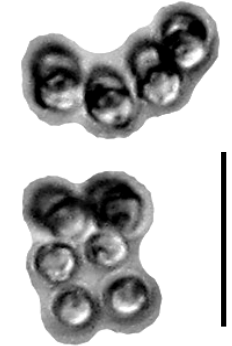

5

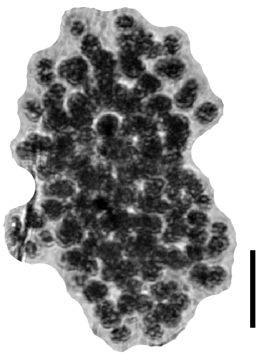

9

Figures 2 à 11 : 2- "Aphanocapsa grevillei ; 3- *Aphanothece castagnei ; 4- Chroococcus dispersus ; 5- Chroococcus minutus ; 6- Chroococcus turgidus ; 7- Eucapsis alpina ; 8*Gloeocapsa itzigsohnii ; 9- Microcystis aeruginosa ; 10- Microcystis holsatica; 11 - Synechocystis aeruginosus. Cyanoprokaryota - Cyanophyceae - Chroococcales

Cyanoprokaryota - Cyanophyceae - Hormogonales

Figures 12 à 14 : 12- Anabaena affinis ; 13- Anabaena spiroides ; 14- Anabaena sp. 


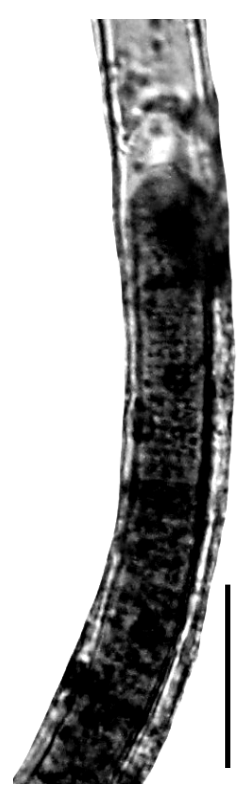

15

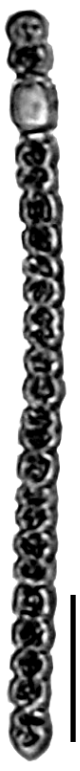

16

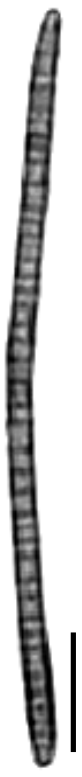

17

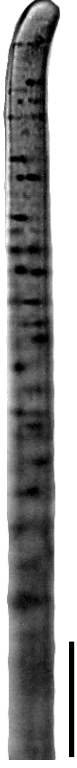

18

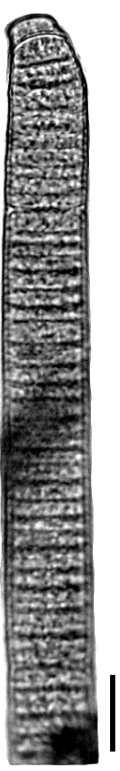

19

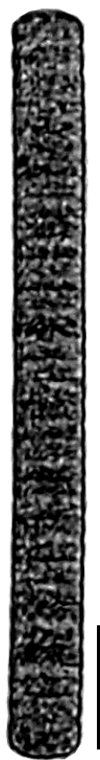

20

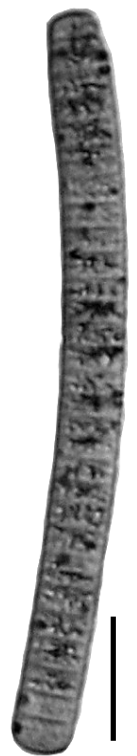

21

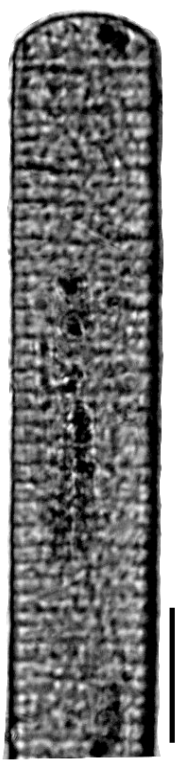

22

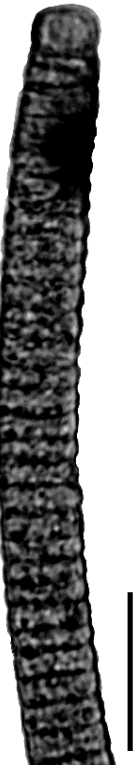

23

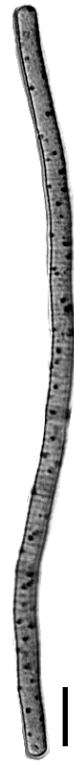

24

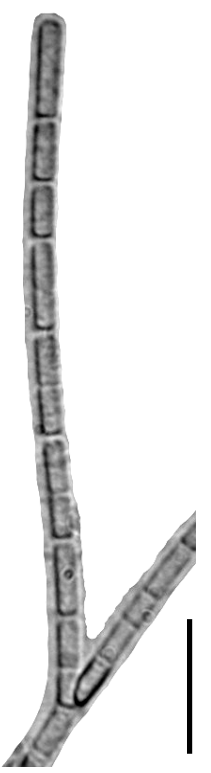

25

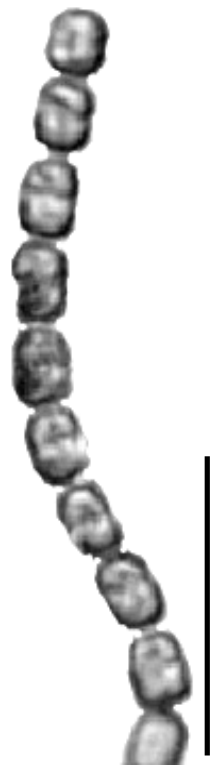

26

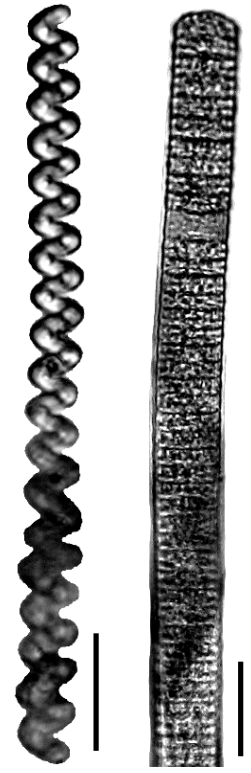

28

Cyanoprokaryota - Cyanophyceae - Hormogonales

Figures 15 à 27 : 15- * Lyngbya major ; 16- Nostoc entophytum ; 17- * Oscillatoria acuta ; 18*Oscillatoria chlorina ; 19- Oscillatoria jenensis ; 20- * Oscillatoria mougeotii ; 21- Oscillatoria ornata, 22- Oscillatoria princeps ; 23-Oscillatoria sancta ; 24- * Phormidium simplissimum ; 25 Plectonema gracillimum ; 26- Pseudanabaena crassa ; 27- Spirulina princeps ; 28- Tychonema bornetii. 
Tableau : Comparaison des travaux exclusifs sur les Cyanoprokaryota de Côte d'Ivoire.

\begin{tabular}{lcc}
\hline & $\begin{array}{c}\text { Bourrelly (1961c), } \\
\text { eaux du Sud }\end{array}$ & $\begin{array}{c}\text { Présente étude, } \\
\text { rivière Boubo }\end{array}$ \\
\hline Nombre total de taxa & 29 & 27 \\
Chroococcales (p.c.) & 31 p.c. & 34,5 p.c. \\
Hormogonales (p.c.) & 69 p.c. & 65,5 p.c. \\
\hline
\end{tabular}

\section{DISCUSSION}

Les récoltes réalisées sur les rivières Boubo et Mé présentent beaucoup d'inégalités non seulement dans la répartition des taxa rencontrés, mais également dans la diversité spécifique. Les récoltes qui proviennent de la rivière Boubo représentent 90 p.c. contre 10 p.c. pour la rivière Mé sur l'ensemble des prélèvements de Cyanoprokaryota. Vingt sept espèces et variétés identifiées sont réparties entre deux ordres; à savoir l'ordre des Chroococcales (37 p.c.) avec sept genres: Aphanocapsa, Aphanothece, Chroococcus, Eucapsis, Gloeocapsa, Microcystis, Synechocystis, et l'orde des Hormogonales (60 p.c.) comportant neuf genres qui sont Anabaena, Lyngbya, Nostoc, Oscillatoria, Phormidium, Plectonema, Pseudanabaena, Spirulina, Tychonema.

La flore Cyanophytique de la rivière Boubo est la plus riche par le nombre de taxa qu'elle renferme. Cette richesse pourrait s'expliquer par le fait que les eaux de cette rivière reçoivent plus de rejets nutritifs provenant du bassin versant et les nombreuses plantations surtout industrielles (Palmeraie, Hévea) qui l'entourent.

Nos résultats, comparés à ceux de Bourrelly (1961), présentent quelques similitudes. Ainsi, en ne tenant compte que de l'abondance des taxa par ordre, nous remarquons une domination des Hormogonales dans les deux travaux sur les Chroococcales (voir tableau). De plus le genre Oscillatoria (10 p.c.) est le genre le plus représentatif des prélèvements chez Bourrelly tout comme dans le présent travail où ce genre constitue 25,9 p.c. de l'ensemble des taxa inventoriés. Cependant, dans l'ensemble, on observe d'énormes diversités spécifiques aussi bien dans l'identification des taxa que dans leur distribution. Cette variation de la composition des algues peut être attribuée soit à l'hétérogénéité des différents cours d'eau, soit tout simplement au fait que les échantillonnages n'ont pas été faits avec la même régularité (Ouattara et al., 2000).

\section{Conclusion}

Les rivières Boubo et Mé, n'ayant jamais fait l'objet de récolte phytoplanctonique auparavant, présentent grâce à cette étude, une richesse floristique algale fort intéressante, surtout au niveau de la rivière Boubo. De plus les Cyanophyceae retenues pour ce travail sont presque toutes cosmopolites. Vingt sept espèces et variétés ont pu être identifiées, dont neuf espèces sont nouvelles pour la Côte d'Ivoire: Aphanocapsa grevillei, Aphanothece castagnei, Gloeocapsa itzigsohnii, Microcystis holsatica, Lyngbya major, Oscillatoria acuta, Oscillatoria chlorina, Oscillatoria mougeotii, Phormidium simplissimum.

Microcystis aeruginosa jugée potentiellement toxique a été observée dans les récoltes effectuées sur la rivière Boubo, de même que dans la plupart des travaux d'identification réalisés en Côte d'Ivoire. Cette présence recurrente de ladite espèce dans certains cours d'eau du pays doit pouvoir justifier la conduite d'éventuelles études sur l'écologie de ce taxon en mettant en place un projet d'observation périodique des microalgues en général, des Cyanoprokaryota en particulier. La recherche d'autres taxa potentiels toxiques par des tests de toxicités simples par voie chimique ou immunologique 
et la surveillance fréquente des écosystèmes aquatiques en fonction des usages doivent être un plus pour le développement durable.

\section{RÉFÉRENCES}

Berger C, Couté A, Ba N, Gugger M. 2005. Cyanobacterial taxa of the Sénégal River system (northern Sénégal, West Africa). Arch. Hydrobiol. Suppl., 159: 147-176.

Bourrelly P. 1961. Cyanophycées de la Côte d'Ivoire. Schweizerische Zeitschrift für Hydrobiologie, 23(21): 209-210.

Cecchi P, Arfi R, Berger C. 2005. Cyanobactéries, potentiel toxique et ressources en eau du Burkina Faso. Rapport de Mission IRD, p.35.

Iltis A. 1982. Peuplements algaux des rivières de Côte d'Ivoire. I. Stations de prélèvement, méthodologie, remarques sur la composition qualitative et biovolumes. Rev. Hydrobiol.Trop., 15(3): 231-239.

Komárek JK, Anagnostidis K. 1999. Cyanoprokaryota 1. Teil : Chroococcales. In Süßwasser Flora von Mitteleuropa, Ettl H, Gärtner G, Heynig H, Mollenhauer D (eds). Gustav Fischer: Jena, Stuttgart, Lübeck ; 1-548.
Komárek JK. Anagnostidis K. 2005. Cyanoprokaryota 2 : Oscillatoriales. In Susswasser Flora von Mitteleuropa, Büdel B, Krienitz L, Gärtner G, Schagerl M (eds). Elsevier/Spektrum: Heidelberg, 1-759.

Niamien JE. 2010. Composition et distribution spatio-temporelle des peuplements d'algues de quatre rivières de Sud-Est de la Côte d'Ivoire (Soumié, Éholié, Éhania et Noé). Thèse Unique de Doctorat, Université d'Abobo-Adjamé, Abidjan, p. 146.

Ouattara A, Podoor N, Teugels GG, Gourène G. 2000. Les micro-algues de deux cours d'eau (Bia et Agnébi) de Côte d'Ivoire. Syst. Geogr. Pl., 70: 315-372.

Roger PA, Reynaud PA. 1977. La biomasse algale dans les rizières du Sénégal : importance relative des Cyanophycées fixatrices de $\mathrm{N}_{2}$. ORSTOM Rev. l'Écol. Biol. Sci., 14(4): 519-530.

Serpette M. 1955. Contribution à l'étude des Cyanophytes de l'Afrique Occidentale. Bull. I.F.A.N., 3: 769-804.

Whittton BA. 1968. Blue-green algae from Sierra Leone. Nova Hedwigia, 15: 203209. 